\title{
Les passes à poissons : méthodes et techniques générales
}

\author{
Fishways : Principles and design criteria
}

\author{
M. Larinier \\ Division "Qualité des eaux, pêche et pisciculture » \\ CEMAGREF, BP 26, Castanet Tolosan Cedex
}

L'auteur fait la synthèse des connaissances servant de base à la conception des dispositifs permettant le franchissement des barrages par les poissons migrateurs. Les principes de fonctionnement, les critères de dimensionnement et les conditions d'utilisation des différents types de passes (passes à bassins successifs, passes à ralentisseurs, ascenseurs et écluses) sont évoqués. L'accent est mis sur l'importance de la situation et de l'attractivité de ces ouvrages qui doivent être à l'échelle de l'aménagement et du cours d'eau.

The writer synthesizes knowledge basically used for constructing devices which enable migrating fish to pass through basin catchments. He describes the dimensioning criteria and utilization conditions for various types of ladders (fish ladders in successive basins, slow-motion passes, lifts and sluices). The emphasis is put on the significance of the situation and attractiveness of these undertakings which must concern the level of improvement and rivers.

\section{Introduction}

On donne le nom de "passes ", "d'échelles ", ou plus généralement de dispositif de franchissement aux systèmes permettant aux poissons de franchir un obstacle. Leur principe consiste à attirer le migrateur en un point déterminé du cours d'eau à l'aval de l'obstacle et à les inciter - voire à les obliger - à passer en amont en leur ouvrant une voie d'eau ou en les piégeant dans une cuve et en déversant celle-ci en amont.
Vitesses et hauteurs de chute dans les passes doivent rester compatibles avec les capacités de nage et de saut des espèces concernées. D'autres paramètres comme la turbulence, l'éclairement, le bruit peuvent influer sur le comportement du poisson.

Certains types de passe sont spécifiques dans la mesure où leur conception et leurs caractéristiques hydrauliques sont adaptées au comportement migratoire bien particulier de certaines espèces (passes à civelles par exemple).

Les dispositifs de franchissement doivent d'autre part éviter les risques de blessure, un stress excessif pour le 


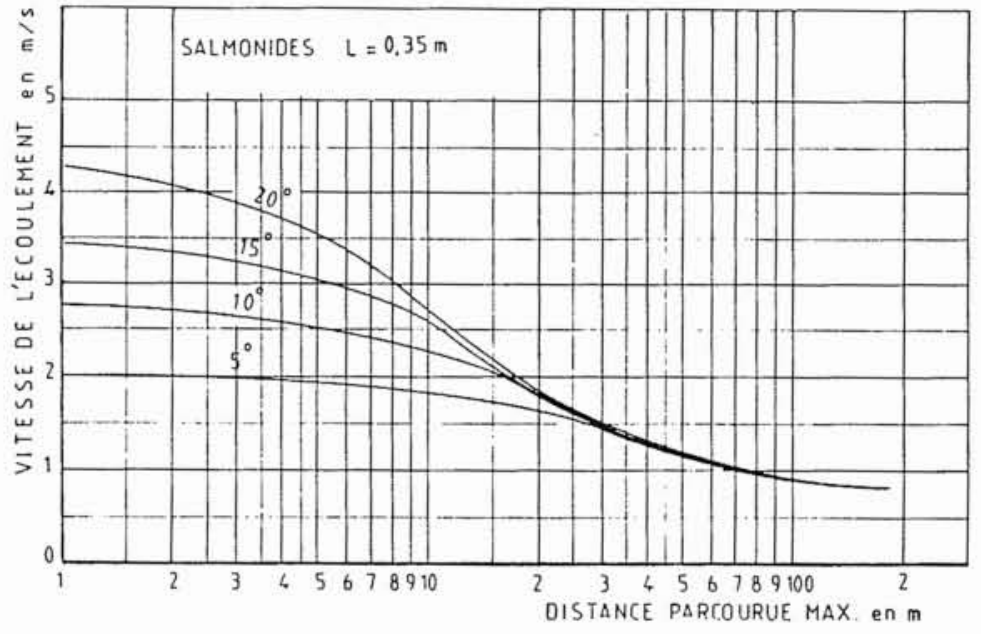

Figure 1. - Distance parcourue maximale en fonction de la vitesse de l'écoulement (salmonidé de $0,35 \mathrm{~m}$ de longueur) d'après Beach, 1984. poisson, les retards dans les migrations et minimiser les problèmes d'entretien et de maintenance.

Il est impossible de passer en revue dans ces quelques pages toutes les techniques de franchissement utilisées, certaines répondant à des situations bien particulières. On n'évoquera que les dispositifs les plus couramment utilisés (passes à bassins successifs, ralentisseurs, etc.), les autres étant d'ailleurs généralement basés sur des principes voisins.

\section{Performances de nage et conditions hydrauli- ques dans les passes}

Un facteur primordial dans la conception des passes à poissons est la prise en compte de la capacité de nage du migrateur, en terme de vitesse de nage et d'endurance.

On distingue fréquemment plusieurs niveaux d'activité de nage (Blaxter, 1969; Bell, 1973; Webb, 1975) sur la base du temps pendant lequel la vitesse peut être maintenue.

- le vitesse " de croisière" (« cruising speed »), vitesse susceptible d'être maintenue pendant des heures par le poisson sans engendrer des modifications physiologiques profondes de son organisme (mécanismes d'activité musculaire aérobies);

- la vitesse "soutenue" (" sustained speed "), vitesse pouvant être maintenue plusieurs minutes mais entraînant la fatigue du poisson;

- la vitesse "de pointe ", ou "de sprint" ("burst speed »), vitesse résultant d'un effort violent ne pouvant être maintenue qu'un temps limité (mécanismes d'activité musculaire anaérobies).

Des études expérimentales (Wardle, 1975; Wardle, 1980) ont permis de dégager des expressions empiriques (Zhou, 1982; Beach, 1984) prédisant les vitesses maximales de nage ainsi que le temps pendant lequel le poisson peut soutenir ces vitesses. Ces formules mettent en évidence le rôle primordial non seulement de la taille du poisson, mais aussi de la température de l'eau, la vitesse maximale de nage d'un individu pouvant être réduite de moitié lorsque la température passe de $18^{\circ} \mathrm{C}$ à $5^{\circ} \mathrm{C}$. On a porté sur la figure 1 les distances maximales susceptibles d'être franchies par un salmonidé de $35 \mathrm{~cm}$ de longueur dans un écoulement de vitesse moyenne donnée.

Il convient cependant de noter :

- que la plupart des poissons sont capables de percevoir de très faibles variations de vitesses et chercheront généralement les zones les plus favorables à leur progression (voisinage des parois, zones de décollement);

- que lorsque les fluctuations spatio-temporelles de vitesses sont intenses (écoulements tourbillonnaires, par exemple), l'énergie nécessaire au migrateur pour franchir une certaine distance peut devenir beaucoup plus importante que celle requise pour parcourir la même distance dans un écoulement plus régulier ayant même vitesse moyenne;

- que les performances de nage du poisson approchant de sa maturité sexuelle sont réduites (Blaxter, 1969), les migrateurs comme le saumon ou l'alose ne se nourrissant plus lorsqu'ils effectuent leur remontée vers les frayères. 
Dans la conception des ouvrages de franchissement, les vitesses de l'eau doivent être généralement maintenues bien au-dessous des vitesses de pointe, dans le domaine des vitesses soutenues et ce, pour les températures les plus faibles susceptibles d'être rencontrées en période de migration et les individus des espèces migratrices concernées les moins vigoureux.

La surestimation des capacités de nage des migrateurs (passes trop " acrobatiques") par les aménageurs est à l'origine de la raréfaction, voire de la disparition de certaines espèces sur de nombreux cours d'eau.

\section{Les passes à bassins successifs}

Le type de passe le plus couramment utilisé est la passe à bassins successifs : la hauteur à franchir est divisée en plusieurs petites chutes formant une série de bassins (figure 2) qui communiquent entre eux par'des déversoirs, des échancrures plus ou moins profondes, des orifices noyés ou des fentes verticales. Le dénivelé entre deux bassins est avant tout fonction des espèces migratrices. On adopte généralement des valeurs comprises entre 30 et $45 \mathrm{~cm}$ pour les salmonidés grands migrateurs, de 15 à $30 \mathrm{~cm}$ pour les autres espèces (Clay, 1961; Jens, 1971; Larinier, 1977, 1983; Conrad et Jansen, 1983; Beach, 1984).

Le débit dans la passe est généralement fonction de l'importance du cours d'eau, pouvant varier de moins de $100 \mathrm{l} / \mathrm{s}$ à plusieurs $\mathrm{m}^{3} / \mathrm{s}$.

La turbulence dans les bassins doit rester à un niveau acceptable pour le migrateur. On admet une puissance dissipée maximale par unité de volume d'eau dans les bassins comprise entre 150 et $200 \mathrm{~W} / \mathrm{m}^{3}$ (Bell, 1973; Larinier, 1977; Conrad et Jansen, 1983). Si la passe ne comporte que quelques bassins, des valeurs sensiblement plus importantes peuvent être adoptées.

La forme des bassins (leur volume étant fixé par le débit et le dénivelé entre bassins) est liée au mode de communication entre bassins (il convient d'éviter le passage direct d'un jet à forte vitesse d'un bassin à l'autre) et quelquefois à des contraintes liées au site. Les longueurs moyennes sont de l'ordre de 2,50 à $3,00 \mathrm{~m}$. Elles peuvent varier, dans des cas exceptionnels de moins de $1,50 \mathrm{~m}$ pour des passes à faible débit à plus de $4,50 \mathrm{~m}$ pour des passes à fort débit. Leur pente peut donc varier de plus de $20 \%$ à moins de $7 \%$. Pour les grands migrateurs, il est préférable de ne pas prendre des tirants d'eau dans les bassins inférieurs à 1-1,2 mètre.

Dans une passe à bassins, le poisson ne fournit d'effort qu'au passage de l'échancrure, de l'orifice ou de la fente sur une distance n'excédant pas quelques décimètres. Le poisson peut, d'autre part, éviter les zones à vitesses maximales en profitant des décollements des lames d'eau.

\section{Les passes à ralentisseurs}

Dans les passes à ralentisseurs - ou Denil - on dispose dans un canal rectiligne des déflecteurs régulièrement espacés, donnant naissance à des courants en hélice qui assurent, par des transferts intenses de quantité de mouvement, une forte dissipation d'énergie au sein de l'écoulement et une réduction importante des vitesses. Les caractéristiques géométriques et hydrauliques d'une vingtaine de ralentisseurs de forme plus ou moins complexes ont été obtenues suite à des études sur modèles réduits (Denil, 1936-38; McLeod et Nemenyi, 1940; White et Nemenyi, 1942, Lachadenede, 1931; Ziemer, 1962; Larinier, 1978; Lonnebjerg, 1980; Larinier et Miralles, 1981; Larinier, 1983).

Les passes à ralentisseurs les plus couramment utilisées, que ce soit en Irlande, sur la Côte Est du Canada et des Etats-Unis, en France et plus récemment en Angleterre sont les passes à ralentisseurs plans (figure 3 ).

Le poisson franchit une passe à ralentisseurs d'une seule traite. Il ne peut se reposer entre les ralentisseurs : lorsque la dénivellation devient importante, le migrateur doit fournir un effort intense et il semble préférable de prévoir des bassins de repos. Les volées des passes à ralentisseurs sont rectilignes et tout changement de direction doit s'effectuer au niveau d'un bassin de repos.

De façon générale, les passes à ralentisseurs - en particulier les passes à ralentisseurs de fond - sont plus sélectives que les passes à bassins successifs et ne conviennent pour la plupart qu'aux migrateurs de grandes tailles.

\section{Ecluses et ascenseurs}

L'écluse à poissons - ou écluse Borland (Aitken et al., 1966) fonctionne suivant un principe voisin de celui d'une écluse de navigation : les migrateurs sont piégés dans un sas, puis éclusés comme le serait un bateau (figure 4).

Le cycle de fonctionnement peut se résumer ainsi : le migrateur est attiré dans la chambre aval, le débit dans l'écluse étant contrôlé par la vanne amont. La vanne aval est fermée, l'écluse est remplie. On incite le poisson à passer dans la retenue amont en établissant un courant d'attrait par l'ouverture d'un by-pass. Au bout d'un certain temps, on procède à la réouverture de la vanne aval et à la vidange de l'écluse.

L'efficacité d'un tel dispositif est liée non seulement à son attractivité, mais aussi au comportement du poisson dans l'écluse qui doit demeurer dans la chambre aval pendant toute la phase d'attrait, suivre le niveau de l'eau

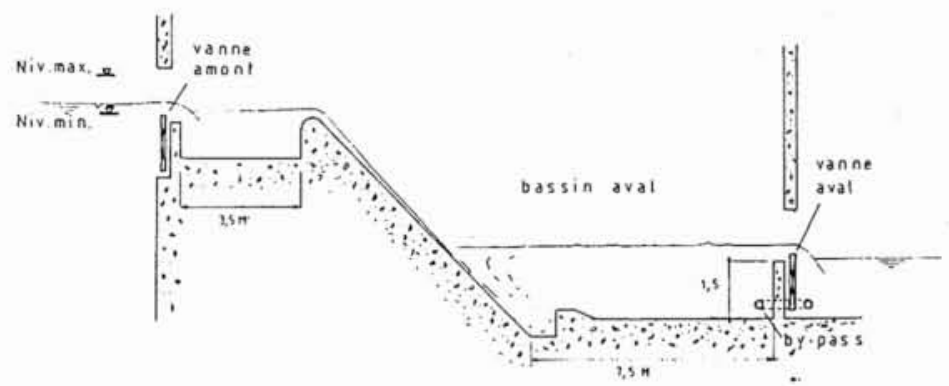

Figure 4. - Schéma de principe d'une écluse à poissons. 


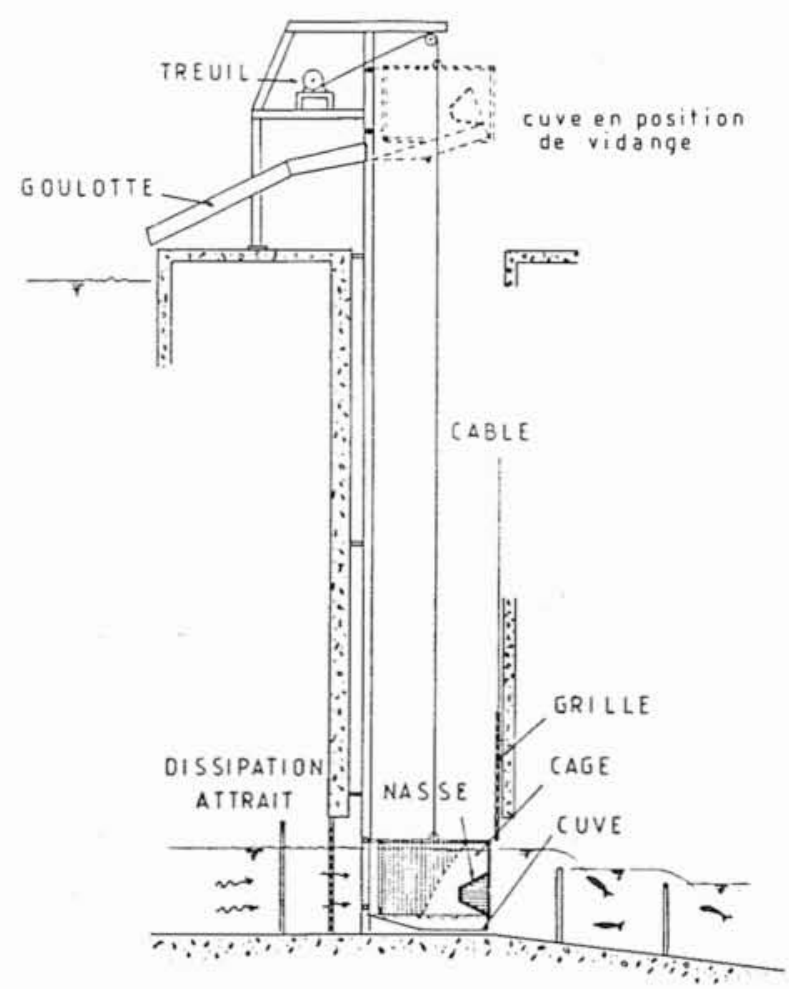

Figure 5. - Schéma de principe d'un ascenseur à poissons.

lors de la phase de remontée et sortir de l'écluse avant la vidange. A cet égard, il est nécessaire que les vitesses et les turbulences dans la chambre restent acceptables pour le migrateur, que ce soit en phase d'attrait ou de remplissage.

Comme il est impossible de déterminer a priori quelles seront les conditions hydrauliques optimales pour les migrateurs, il convient de donner au fonctionnement de l'écluse un maximum de souplesse (dans la durée de chaque phase du cycle, dans le temps et le degré d'ouverture des différentes vannes et du by-pass).

Malgré ces précautions - et a fortiori quand elles n'ont pas été prises - de nombreuses écluses se sont révélées peu ou pas efficaces, en particulier en France.

Le principe de l'ascenseur consiste à piéger les migrateurs dans une cuve au pied de l'obstacle et à déverser celle-ci soit directement dans la retenue amont, soit dans un canal communiquant avec le bief amont. Les migrateurs peuvent être directement piégés dans une cuve, un dispositif anti-retour (système de nasse) les empêchant d'en ressortir. On a porté sur la figure 5 le principe de l'ascenseur récemment mis en service au barrage de Poutès sur l'Allier.

Lorsque les pics de migration sont importants (en particulier pour l'alose), on piège dans un premier temps les poissons dans un vaste bassin de stabulation, puis on les concentre au-dessus de la cuve de l'ascenseur en les poussant à l'aide d'une grille montée sur un charriot mobile (ascenseurs utilisés sur la Côte Est des Etats-Unis - Rizzo, 1969 - ascenseur à l'usine de Golfech sur la
Garonne). Les volumes de la cuve et du bassin de stabulation sont déterminés en fonction de l'importance du pic de migration (Bell, 1973; Travade, 1982).

\section{Choix du type de dispositif de franchissement}

De nombreux facteurs ou contraintes d'ordre biologique, hydrologique, hydraulique, topographique interviennent dans le choix et le dimensionnement d'un dispositif de franchissement. Citons en particulier :

- les espèces migratrices présentes dans les cours d'eau; - les caractéristiques hydrologiques du cours d'eau;

- les cotes des plans d'eau amont et aval ainsi que leurs variations en période de migration;

- la configuration de l'obstacle et la topographie du lit à l'aval;

- la gestion de l'eau.

Lorsqu'on a affaire à plusieurs espèces migratrices (grands migrateurs, migrateurs holobiotiques), la passe à bassins successifs est la meilleure solution, moins sélective que la plupart des passes à ralentisseurs. La passe à orifices noyés présente généralement peu d'intérêt. Les passes à fentes verticales (à une ou deux fentes suivant l'importance du débit) ne conviennent que pour des gros ouvrages (débits de l'ordre de $1 \mathrm{~m}^{3} / \mathrm{s}$ au minimum). Les passes à bassins à échancrures déversantes, associées ou non à des orifices de fond peuvent s'adapter à de nombreux cas de figure : débits de quelques dizaines de litres $/ \mathrm{s}$ à plusieurs $\mathrm{m}^{3} / \mathrm{s}$, et dans la mesure où les échancrures sont suffisamment profondes, à des variations du niveau amont relativement importantes.

Les passes à ralentisseurs présentent un intérêt particulier pour le saumon et la truite de mer, pour les obstacles de hauteur modérée et pour les ouvrages existants, dans la mesure où il est beaucoup plus facile d'intégrer ce type de passe dans le corps d'un barrage qu'une passe à bassins successifs dimensionnée pour un débit identique. D'où sa fréquente utilisation sur les petites rivières à saumons bretonnes.

Lorsque la hauteur de l'obstacle à franchir devient plus importante, la nécessité de prévoir des bassins de repos augmente la longueur du dispositif et peut rendre aussi intéressante l'installation d'une passe à bassins successifs.

L'inconvénient majeur des passes à ralentisseurs réside dans le fait que leur calage exige une connaissance très précise des niveaux d'eau amont et aval, ce qui n'est pas toujours possible (modification des lignes d'eau suite à l'installation de l'ouvrage dans le lit ou à des extractions de matériaux alluvionnaires en aval).

Pour les ouvrages de hauteurs importantes, l'installation d'un ascenseur ou d'une écluse est plus facile et beaucoup moins coûteuse que celle d'une passe classique. La présence de nombreux organes mobiles - vannes, cuves, grilles - se traduit cependant par des coûts de maintenance élevés. C'est la raison pour laquelle on peut leur préférer dans certains cas des passes "statiques" d'un génie civil plus coûteux mais d'un entretien moins lourd et d'une fonctionnement plus fiable.

Un ascenseur est généralement préférable à une écluse : le problème de l'attractivité est identique, mais les pois- 
sons piégés dans la cuve de l'ascenseur passeront obligatoirement à l'amont, ce qui n'est pas forcément le cas pour les poissons piégés dans la chambre aval d'une écluse Borland.

\section{L'attractivité des passes : Implantation et conditions hydrodynamiques aux entrées}

Les migrateurs doivent trouver l'entrée de la passe le plus rapidement possible lorsqu'ils arrivent sur l'obstacle. L'attractivité d'un dispositif de franchissement est liée à la situation de l'entrée - entrée pour le poisson, donc partie aval - et aux conditions hydrodynamiques (débits, vitesses, lignes de courant) au voisinage de cette entrée qui ne doit être masquée ni par des écoulements provenant des turbines ou d'ouvrages évacuateurs, ni par des zones de recirculation ou d'eaux mortes

Le débit du dispositif de franchissement doit être à l'échelle des débits du cours d'eau en période de migration (plusieurs pour cent). Si une passe de quelques centaines de litres secondes peut se révéler suffisamment attractive sur une petite rivière bretonne, des dispositifs de franchissement sur des cours d'eau comme la Dordogne, la Garonne ou la Loire nécessiteront des débits de plusieurs $\mathrm{m}^{3} / \mathrm{s}$. Pour limiter le génie civil de l'ouvrage, il est possible de ne faire transiter qu'une partie du débit dans la passe, le débit complémentaire nécessaire à l'attraction étant injecté à très faible vitesse dans le tronçon aval de la passe ou à l'entrée même du dispositif.

Pour les grands migrateurs - truite de mer, saumon, alose - l'expérience montre qu'il est indispensable de créer au niveau de l'entrée des vitesses élevées, vitesses devant cependant rester compatibles avec la capacité de nage de toutes les espèces migratrices concernées. Des valeurs de 1,5 à $2,5 \mathrm{~m} / \mathrm{s}$ sont communément admises.

Un soin particulier doit être apporté à la conception de l'entrée d'une passe lors de l'élaboration d'un projet, en particulier sur les grands cours d'eau : on s'assurera que les vitesses y restent suffisamment élevées pour les différentes conditions du niveau aval susceptibles d'être rencontrées en période de migration. Des vitesses suffisantes peuvent être maintenues soit en agissant sur les sections d'écoulement (en "pinçant" plus ou moins l'écoulement à l'aide d'une vanne mobile), soit en modulant le débit dans l'ouvrage suivant les conditions de niveau d'eau à l'aval de l'obstacle.

Pour un barrage existant - ou un obstacle naturel l'observation du comportement du migrateur (route de migration, zones de stabulation, points du barrage où s'effectuent les tentatives de franchissement) peut aider à choisir la situation de l'entrée de la passe.

Dans le cas d'un ouvrage en projet ou en l'absence de migrateurs (programmes de restauration), on ne peut faire que des hypothèses sur le comportement du poisson.

De façon générale, le poisson a tendance à remonter dans le courant le plus à l'amont possible, jusqu'à ce qu'il soit arrêté par une chute d'une hauteur infranchissable ou par des courants ou turbulences trop violents. Il convient donc d'installer la passe le plus près possible du point ou de la ligne de plus haute remontée du migrateur.
Lorsque l'obstacle est implanté perpendiculairement à l'axe de l'écoulement, la manière dont le poisson se présentera sur l'obstacle dépend de la répartition des débits sur l'ouvrage et de la topographie du lit à l'aval. Sur un obstacle de forte largeur, deux passes pourront se révéler nécessaires. Dans le cas d'un barrage équipé de bouchures mobiles, il est possible de régler le déversement de façon à guider le migrateur vers l'entrée des passes.

Dans le cas d'un aménagement hydroélectrique, lorsque tout le débit est turbiné, les migrateurs seront généralement attirés vers les aspirateurs des turbines. Il conviendra donc d'installer l'entrée de la passe du côté de l'usine. Par contre, lorsque les ouvrages évacuateurs déversent, le migrateur pourra se présenter soit du côté de l'usine, au pied des turbines, soit du côté du barrage : on peut être amené à envisager deux dispositifs de franchissement.

Le comportement du migrateur n'est pas le seul facteur à considérer dans le choix de l'implantation de la passe : il convient de prendre en compte l'exposition de la passe aux crues, sa protection contre les corps flottants et son accessibilité de façon à assurer sa surveillance, son contrôle et son entretien. A cet égard, les passes situées en rive ou à proximité d'une rive sont préférables à celles implantées dans le corps même d'un déversoir ou d'un seuil.

La protection des passes contre les corps dérivants s'effectue par les moyens classiques employés sur les prises d'eau des ouvrages hydrauliques : drômes flottantes, masques de surface, grilles à barreaux suffisamment espacés pour permettre le passage du migrateur, écrans en maçonnerie ou en béton, rangées de pieux battus à l'amont de l'échelle.

La sortie de la passe - sortie pour le poisson, donc partie amont - ne doit se trouver ni dans une zone de forte vitesse, à proximité d'un ouvrage évacuateur afin que le poisson ne risque pas d'être entrainé de nouveau à l'aval, ni dans une zone d'eau morte ou de recirculation dans laquelle le poisson peut se trouver piégé. Dans le cas d'une usine hydroélectrique, la passe à poissons, si sa sortie est convenablement placée par rapport à la prise d'eau, pourra être utilisée comme dispositif de dévalaison.

Chaque fois que cela sera possible, un emplacement permettant l'installation d'un dispositif de contrôle (compteur, piège) sera réservé dans la partie amont de la passe.

\section{Conclusion}

La conception d'une passe à poissons qui relève à la fois de l'hydrotechnique et de la biologie, est souvent délicate. Les plans du dispositif doivent être poussés dans les moindres détails et il est indispensable de suivre de très près l'exécution des travaux : toute improvisation consécutive à un manque de précision des plans peut rendre le dispositif partiellement ou totalement inefficace.

La mise au point d'un dispositif de franchissement efficace peut demander plusieurs années après sa construction. Il est donc important de concevoir un système très souple permettant un certain nombre d'ajustements ou de modifications. Il ne faut cependant pas oublier que l'établissement d'une passe à poissons n'est 
qu'un pis aller : l'efficacité d'une passe est rarement totale et tout obstacle, principalement sur les grands cours d'eau, même équipé d'ouvrages de franchissement bien conçus, induit, dans le meilleur des cas, des retards dans les migrations : il suffit de prendre pour exemple les ouvrages situés sur la rivière Columbia aux Etats-Unis dont les passes à poissons monumentales servent cependant souvent de référence dans le domaine (Larinier, Travade, 1982). Il convient donc avant tout d'éviter la construction de nouveaux barrages sur les grands axes de migration.

\section{Bibliographie}

Aitken P.L., Dickerson L.H., Menzies W.J.M., 1966. - Fish passes and screens at water power works. Proc. Instn. Ci. Engrs. 35, Sept. 29-57.

BEACH M.H., 1984. - Fish pass design - criteria for the design and approval of fish passes and other structures to facilitate the passage of migratory fish in rivers - Fisheries Research Technical Report $-\mathrm{N}^{\circ} 78-$ Ministry of Agriculture, Fisheries and Food - 46 pp.

BELL M.C., 1973. - Fisheries handbook of engineering requirements and biological criteria. Fisheries Engineering Research Program. U.S. Army Corps of Engineers, North Pacific Division. Portland, Oregon.

BLAXTER J.H.S., 1969. - Swimming speeds of fish. FAO Fish. Rep, $62(2): 69-100$

CLAY C.H., 1961. - Design of fishways and other fish facilities. Ottawa, Department of Fisheries of Canada, 301 p.

ConRad V., Jansen H., 1983. - Refinements in Design of Fishways for small Watersheds Northeast Fish and Wildlife Conference. Dover, Vermont, May, $26 \mathrm{p}$.

DENIL G., 1936-1938. - La mécanique du poisson de rivière. Ann. Trav. Publ. Belg., août et octobre 1936, février à décembre 1937, février à août 1938, $395 \mathrm{p}$.

Jens G., 1971. - Funktion, Bau und Betrieb von Fischpässen. Arch. Fisch. Wiss., 22, Beih. 1, 27 p.

LaChadenede B., 1931. - Echelle à poissons. Rev. Eaux For., n 9 septembre 1931: 763-770.

LARINIER M., 1977. - Les passes à poissons. C.T.G.R.E.F. Division Qualité des Eaux, Pêche et Pisciculture, Etude $n^{\circ} 16$, $126 \mathrm{p}$.

LARINIER M., 1978. - Etude du fonctionnement d'une passe à poissons à ralentisseurs plans. Bull. Fr. Pisc., 271: 40-54.

LARINIER M., MIRAlles A., 1981. - Caractéristiques hydrauliques des passes à ralentisseurs - CEMAGREF-DQEPP - rapport non publié $-46 \mathrm{p}$.

LARINIER M., TRAVADE F., 1982. - Les poissons migrateurs aux
Etats-Unis. Ouvrages de franchissement de barrages. Programmes de restauration des populations. E.D.F. Direction des Etudes et Recherches. CEMAGREF. Division Qualité des Eaux, Pêche et Pisciculture, 73 p.

LARINIER M., 1983. - Guide pour la conception des dispositifs de franchissement des barrages pour les poissons migrateurs. Bull. Fr. Pisc., numéro spécial, 39 pp.

LONNEBJERG N., 1980. - Fiskepas af modstromstypen. (Fishways of the Denil type) Meddr Ferskvandsfiskerilab. Danm. Fisk. -og Havunders., Silkeborg, (1) 1980, 107 pp.

MCLEOD A.M. and NEMENYI P., 1940. - An investigation of fishways. Bull. Iowa St. Univ., Ames, lowa, 24 : 1-72.

Rızzo B., 1969. - Fish passage facilities design parameters for Connecticut river dams. Turners Falls dam. Bureau of sport Fisheries and Wildlife, Boston, Massachussetts, 33 p.

TRAVADE F., 1982. - Ascenseur à poissons. Critẻres de conception. Application au barrage de Golfech - E.D.F. Direction des Etudes et Recherches. Département Environnement Aquatique et Atmosphérique. HE/31 - 82-48, 36 p.

WARDLE C.S., 1975. - Limit of fish swimming speed. Nature, Lond., $255:$ 725-727.

WARDLE C.S., 1980. - Effects of temperature on the maximum swimming speed of fishes. pp. 519-531 in Environmental Physiology of Fishes. Plenum Press, New York and London, $723 \mathrm{pp}$.

WEBB P.W., 1975. - Hydrodynamics and energetics of fish propulsion. Bull. Fish. Res. Bd Can., (190), 1-158.

WhIte C.M., NeMENYI P., 1942. - Report of Hydraulic Research on fish passes, in Report of the Committee of fish passes. Instn. civ. Engrs, London, 59 pp.

ZHOU Y., 1982. - The swimming behaviour of fish in towed gears; a reexamination of the principles. Scott. Fish. Work. Pap., Dept. Agric. Fish. Scotl., (4), 1-55.

ZIEMER G.L., 1962. - Steeppass fishway development. Inf. Leafl., Alaska Dept. Fish. Game, Div. Engng Serv., (12), 1-32. 
M. TOULLIC. - Cette question concerne principalement E.D.F. Alors que la production d'électricité est suffisante et que les centrales nucléaires tournent au ralenti, pourquoi E.D.F. continue-t-elle à acheter le courant aux propriétaires de microcentrales dont l'existence est dommageable à la vie piscicole ?

M. WEISROCK. - E.D.F. a une obligation légale d'achat du courant de ces micro-centrales, mais la récente loi sur la pêche dispose que cette obligation est suspendue si l'Administration signale à E.D.F. que l'exploitant est en contravention avec la réglementation sur l'environnement.

M. MiCHon. - Peut-on construire des passes qui arrêtent sélectivement les espèces indésirables ?

M. LARINIER. - Le transit des poissons dans une passe est avant tout fonction de leur capacité de nage. De façon schématique, pour une espèce donnée, les individus les plus grands sont capables de nager plus vite et plus longtemps. Par conséquent, il serait possible de sélectionner la taille des indivïaus capables de franchir une passe en jouant sur la vitesse de l'écoulement. Par contre, il ne parait guère possible de sélectionner les espèces sauf peut-être les quelques espèces ayant un comportement migratoire bien particulier (alose par exemple).

M. PICARD. - Les exemples donnés par M. LARINIER concernent des barrages à niveau amont constant ou faiblement variable. Que peut-on faire dans le cas d'un barrage à niveau amont fortement variable (par exemple un barrage de soutien d'étiage)?

M. LARINIER. - Pour des hauteurs de marnage de l'ordre de 3 à $4 \mathrm{~m}$, on peut envisager des passes à fentes verticales; au-delà de 3 ou $4 \mathrm{~m}$ de marnage, il semble que l'ascenseur s'impose.

M. PICARD. - Une écluse de navigation est-elle efficace pour le transit des migrateurs?

M. LARINIER, - Les écluses de navigation sont rarement utilisées pour assurer le transit des migrateurs, à cause de leur manque d'attractivité. Des expérimentations effectuées en U.R.S.S. ont montré que des écluses de navigation pouvaient se révéler efficaces, à condition d'y faire transiter des débits importants.

M. CUINAT - Les écluses peuvent être très efficaces pour la descente des smolts, surtout si on les manœuvre fréquemment la nuit, avec un éclairage artificiel, en période de dévalaison de ces poissons.

M. PICARD. - En ce qui concerne le barrage de Vichy, pourrait-on supprimer la période d'abaissement du barrage en lâchant un débit réservé de $10 \mathrm{~m}^{3} / \mathrm{s}$ et en construisant une passe à poissons mieux conçue ?

M. LARINIER. - Les problèmes ne sont pas les mêmes sur les grands et les petits cours d'eau. A Vichy, nous avons affaire à un grand cours d'eau. Le problème qui se pose est celui de l'attractivité de la passe, le barrage ayant une longueur supérieure à $100 \mathrm{~m}$. Le migrateur peut se présenter en n'importe quel point du barrage et il mettra un certain temps pour trouver l'entrée de la passe. Même si d'un point de vue hydraulique, les dispositifs de franchissement peuvent être considérés comme efficaces, le barrage induira toujours un certain retard dans la migration du poisson. Dans le cas de Vichy, on constate d'autre part que l'accès aux passes actuelles est rendu plus difficile suite à l'abaissement du lit à l'aval.

M. PICARD. - Sur l'Allier, pourquoi ne fait-on rien au pont du Guetin dont le radier est un obstacle à la remontée ?

M. CUINAT - Le pont du Guetin (pont-canal proche du Bec d'Allier) constitue un obstacle de plus en plus difficile à mesure que le lit se creuse avec les extractions de matériaux : la chute est passée en 10 ans de $50 \mathrm{~cm}$ à $1,10 \mathrm{~m}$.

Il y a deux ans on a constaté un blocage des saumons en période de faible débit et basse température.

Pour éviter que le problème ne se pose à nouveau, il est nécessaire de réaliser deux passes à poissons (au moins une dans une première tranche de travaux) sur cet obstacle.

Toutefois, ce seuil est situé sur le territoire de deux autres délégations du C.S.P., et surtout il y a conflit entre l'intérêt général (faciliter la montée vers les frayères) et l'intérêt particulier des pêcheurs locaux qui voient leurs prises augmenter quand les saumons sont ralentis par l'obstacle.

Le coût de la passe projetée (en rive gauche) était de $150 \mathrm{kF}$ environ en 1984

M. LARINIER n'a pas indiqué sa préférence entre les différents types de passes à poissons. A mon avis, les pré-barrages partiels à l'aval d'un obstacle constituent souvent la meilleure solution, car ils sont toujours alimentés en eau proportionnellement au débit de la rivière, donc bien attractifs, et ils ne nécessitent que peu d'entretien.

M. LARINIER. - La meilleure solution est de transformer toute la rivière en passe à poissons comme cela se voit au Canada pour certains radiers de pont qui sont aménagés en chutes successives ne dépassant pas 35 à $40 \mathrm{~cm}$. Mais cette solution n'est possible que pour des chutes ne dépassant pas 2 à $3 \mathrm{~m}$. 